\title{
Discrete Dirac systems on the semiaxis: rational reflection coefficients and Weyl functions
}

\author{
B. Fritzsche, B. Kirstein, I.Ya. Roitberg and A.L. Sakhnovich
}

\begin{abstract}
We consider the cases of the self-adjoint and skew-self-adjoint discrete Dirac systems, obtain explicit expressions for reflection coefficients and show that rational reflection coefficients and Weyl functions coincide.
\end{abstract}

$\operatorname{MSC}(2010): 39 \mathrm{~A} 10,39 \mathrm{~A} 12,47 \mathrm{~A} 40$

Keywords: Discrete self-adjoint Dirac system, discrete skew-self-adjoint Dirac system, Weyl function, reflection coefficient, Bäcklund-Darboux transformation.

\section{Introduction}

Discrete self-adjoint and skew-self-adjoint Dirac systems play an essential role in the study of Toeplitz matrices (and corresponding measures), of discrete integrable nonlinear equations (including isotropic Heisenberg magnet model) and of spectral theory of difference equations (see, e.g., [5, 6, 14, 20, 22] and references therein). Weyl-Titchmarsh theory of discrete systems is actively studied (see, e.g., [3, 13, 27, 28] and various references therein). In particular, Weyl-Titchmarsh theory of discrete self-adjoint and skew-self-adjoint 
Dirac systems was studied in [6, 8, 14, 18,24] (see also references therein). It is known that Weyl-Titchmarsh (or simply Weyl) functions of continuous Dirac systems on the semi-axis are closely related to the scattering data. Some particular results for the self-adjoint systems are contained, for instance, in [1,11] and the general cases of continuous self-adjoint and skew-self-adjoint systems were treated in the recent paper [23]. The present article may be considered as the continuation of the paper [23], where the important discrete case is dealt with. We consider the cases of the self-adjoint and skew-self-adjoint discrete Dirac systems, obtain explicit expressions for reflection coefficients and show that rational reflection coefficients and Weyl functions coincide.

General-type discrete self-adjoint Dirac system has the form:

$$
y_{k+1}(z)=\left(I_{m}+\mathrm{i} z j C_{k}\right) y_{k}(z) \quad\left(k \in \mathbb{N}_{0}\right),
$$

where $\mathbb{N}_{0}$ stands for the set of non-negative integers, $I_{m}$ is the $m \times m$ identity matrix, " $\mathrm{i}$ " is the imaginary unit $\left(\mathrm{i}^{2}=-1\right)$ and the $m \times m$ matrices $C_{k}$ are positive and $j$-unitary:

$C_{k}>0, \quad C_{k} j C_{k}=j, \quad j:=\left[\begin{array}{cc}I_{m_{1}} & 0 \\ 0 & -I_{m_{2}}\end{array}\right] \quad\left(m_{1}+m_{2}=m ; m_{1}, m_{2}>0\right)$.

We introduce the Jost solution and reflection coefficient of the system (1.1), (1.2) in a similar to the continuous case way. Namely, the Jost solution $\left\{F_{k}(z)\right\}(z \in \mathbb{R})$ of the Dirac system (1.1), (1.2) is defined via its asymptotics

$$
F_{k}(z)=\left(I_{m}+\mathrm{i} z j\right)^{k}\left(I_{m}+o(1)\right), \quad k \rightarrow \infty .
$$

The reflection coefficient $\mathcal{R}(z)$ is introduced via the blocks of $F_{0}(z)$ :

$$
\mathcal{R}(z)=\left[\begin{array}{ll}
I_{m_{1}} & 0
\end{array}\right] F_{0}(z)\left[\begin{array}{c}
0 \\
I_{m_{2}}
\end{array}\right]\left(\left[\begin{array}{ll}
0 & I_{m_{2}}
\end{array}\right] F_{0}(z)\left[\begin{array}{c}
0 \\
I_{m_{2}}
\end{array}\right]\right)^{-1} .
$$

Discrete skew-self-adjoint Dirac system (SkDDS) is given (see [8, 14]) by the formula:

$$
y_{k+1}(z)=\left(I_{m}+\frac{\mathrm{i}}{z} C_{k}\right) y_{k}(z), \quad C_{k}=U_{k}^{*} j U_{k} \quad\left(k \in \mathbb{N}_{0}\right),
$$


where the matrices $U_{k}$ are unitary and $j$ is defined in (1.2).

Direct and inverse problems (in terms of Weyl functions) were solved for systems (1.1), (1.2) in [7, 18] and for systems (1.5) in [6,8]. In particular, in the case of rational Weyl matrix functions, direct and inverse problems were solved explicitly using our GBDT version [19,21,24] of the Bäcklund-Darboux transformation. For various versions of Bäcklund-Darboux transformations and related commutation methods see, for instance, [2, 4, 9, 12, 15, 17] and references therein.

The results of the paper imply that the procedures to recover systems from the Weyl functions enable us to recover systems from the reflection coefficients as well. In the next section, we give some preliminary definitions and results in order to make the paper self-sufficient. Two subsections of Section 3 are dedicated to the reflection coefficients in the self-adjoint and skew-self-adjoint cases.

In the paper, $\mathbb{N}$ denotes the set of natural numbers, $\mathbb{R}$ denotes the real axis, $\mathbb{C}$ stands for the complex plane, and $\mathbb{C}_{+}\left(\mathbb{C}_{-}\right)$stands for the open upper (lower) half-plane. The spectrum of a square matrix $A$ is denoted by $\sigma(A)$.

\section{Preliminaries}

\subsection{Self-adjoint case}

1. Self-adjoint discrete Dirac system and stability of the explicit procedure to recover it from the Weyl function was studied in our recent paper [18]. We refer to [18] for the preliminary definitions and results in this subsection. The fundamental $m \times m$ solution $\left\{W_{k}\right\}$ of (1.1) is normalized by

$$
W_{0}(z)=I_{m} .
$$

Definition 2.1 The Weyl function of the Dirac system (1.1) (which is given on the semi-axis $0 \leq k<\infty$ and satisfies (1.2)) is an $m_{1} \times m_{2}$ matrix function 
$\varphi(z)$ in the lower half-plane, such that the following inequalities hold:

$$
\begin{aligned}
& \sum_{k=0}^{\infty} q(z)^{k}\left[\varphi(z)^{*} \quad I_{m_{2}}\right] W_{k}(z)^{*} C_{k} W_{k}(z)\left[\begin{array}{c}
\varphi(z) \\
I_{m_{2}}
\end{array}\right]<\infty \quad\left(z \in \mathbb{C}_{-}\right), \\
& q(z):=\left(1+|z|^{2}\right)^{-1} .
\end{aligned}
$$

(For the case $z \in \mathbb{C}_{+}$, the definition of the Weyl function $\varphi(z)$ of Dirac system (1.1), (1.2) was given in [7].)

2. In order to consider the case of rational Weyl functions, we introduce generalized Bäcklund-Darboux transformation (GBDT) of the discrete selfadjoint Dirac systems. Each GBDT of the initial discrete Dirac system is determined by a triple $\left\{A, S_{0}, \Pi_{0}\right\}$ of parameter matrices. Here, we take a trivial initial system and choose $n \in \mathbb{N}$, two $n \times n$ parameter matrices $A$ $(\operatorname{det} A \neq 0)$ and $S_{0}>0$, and an $n \times m$ parameter matrix $\Pi_{0}$ such that

$$
A S_{0}-S_{0} A^{*}=\mathrm{i} \Pi_{0} j \Pi_{0}^{*} .
$$

Define the sequences $\left\{\Pi_{r}\right\}$ and $\left\{S_{r}\right\}(r \geq 0)$ using the triple $\left\{A, S_{0}, \Pi_{0}\right\}$ and recursive relations

$$
\begin{aligned}
& \Pi_{k+1}=\Pi_{k}+\mathrm{i} A^{-1} \Pi_{k} j \quad(k \geq 0), \\
& S_{k+1}=S_{k}+A^{-1} S_{k}\left(A^{*}\right)^{-1}+A^{-1} \Pi_{k} \Pi_{k}^{*}\left(A^{*}\right)^{-1} \quad(k \geq 0) .
\end{aligned}
$$

From (2.4) $-(2.6)$, the validity of the matrix identity

$$
A S_{r}-S_{r} A^{*}=\mathrm{i} \Pi_{r} j \Pi_{r}^{*} \quad(r \geq 0)
$$

follows by induction. In the self-adjoint case, we introduce admissible triples $\left\{A, S_{0}, \Pi_{0}\right\}$ in the following way.

Definition 2.2 The triple $\left\{A, S_{0}, \Pi_{0}\right\}$, where $\operatorname{det} A \neq 0, S_{0}>0$ and (2.4) holds, is called admissible.

In view of (2.6), for the admissible triple we have $S_{r}>0(r \geq 0)$. Thus, the sequence (potential) $\left\{C_{k}\right\}(k \geq 0)$ is well-defined by the equality

$$
C_{k}:=I_{m}+\Pi_{k}^{*} S_{k}^{-1} \Pi_{k}-\Pi_{k+1}^{*} S_{k+1}^{-1} \Pi_{k+1} .
$$


Moreover, from [18, Theorem 2.5] we see that the matrices $C_{k}$ satisfy (1.2). We say that the potential $\left\{C_{k}\right\}$ is determined by the admissible triple. The potential determined by an admissible triple $\left\{A, S_{0}, \Pi_{0}\right\}$ is called pseudoexponential. We note that the notion of the pseudo-exponential (and strictly pseudo-exponential) potentials for the self-adjoint continuous case was introduced first in [10] (see also [11]). In the discrete case, some additional requirements on the admissible and strongly admissible triples (which determine pseudo-exponential and strictly pseudo-exponential, respectively, potentials) appear.

All Weyl functions $\varphi(z)$ are contractive in $\mathbb{C}_{-}$and all the potentials $\left\{C_{k}\right\}$, such that $\varphi(z)$ (for the corresponding systems) are contractive and $\varphi(-1 / z)$ are strictly proper rational, are determined by some admissible triples [18]. Strongly admissible triples for the self-adjoint case are considered in Subsection 3.1 .

We will need also the matrix function $w_{A}$, which for each $k \geq 0$ is a so called transfer matrix function in Lev Sakhnovich form [24-26] and is defined by the relation

$$
w_{A}(k, \lambda):=I_{m}-\mathrm{i} j \Pi_{k}^{*} S_{k}^{-1}\left(A-\lambda I_{n}\right)^{-1} \Pi_{k} .
$$

The fundamental solution $\left\{W_{k}\right\}$ of the Dirac system (1.1) admits the representation

$$
W_{k}(z)=w_{A}(k,-1 / z)\left(I_{m}+\mathrm{i} z j\right)^{k} w_{A}(0,-1 / z)^{-1} \quad(k \geq 0),
$$

where $w_{A}$ is defined in (2.9) .

Now, we partition $\Pi_{k}$ and write it down in the form

$$
\Pi_{k}=\left[\begin{array}{ll}
\left(I_{n}+\mathrm{i} A^{-1}\right)^{k} \vartheta_{1} & \left(I_{n}-\mathrm{i} A^{-1}\right)^{k} \vartheta_{2}
\end{array}\right],
$$

where $\vartheta_{1}$ and $\vartheta_{2}$ are $n \times m_{1}$ and $n \times m_{2}$, respectively, blocks of $\Pi_{0}$. Assume further in this subsection that

$$
\pm \mathrm{i} \notin \sigma(A) \text {. }
$$

In view of (2.7) and (2.11), setting

$$
R_{r}:=\left(I_{n}+\mathrm{i} A^{-1}\right)^{-r} S_{r}\left(I_{n}-\mathrm{i}\left(A^{*}\right)^{-1}\right)^{-r}
$$


we have

$$
\begin{aligned}
R_{k+1}-R_{k}= & 2\left(I_{n}+\mathrm{i} A^{-1}\right)^{-k-1} A^{-1}\left(I_{n}-\mathrm{i} A^{-1}\right)^{k} \vartheta_{2} \vartheta_{2}^{*}\left(\left(I_{n}-\mathrm{i} A^{-1}\right)^{k}\right)^{*}\left(A^{-1}\right)^{*} \\
& \times\left(\left(I_{n}+\mathrm{i} A^{-1}\right)^{-k-1}\right)^{*} \geq 0 .
\end{aligned}
$$

Since $R_{0}=S_{0}>0$, relations (2.14) imply that there is a limit

$$
\lim _{k \rightarrow \infty} R_{k}^{-1}=\varkappa_{R} \geq 0 .
$$

In a similar way we introduce the matrices

$$
Q_{r}:=\left(I_{n}-\mathrm{i} A^{-1}\right)^{-r} S_{r}\left(I_{n}+\mathrm{i}\left(A^{*}\right)^{-1}\right)^{-r},
$$

and show that

$$
Q_{k+1}-Q_{k} \geq 0
$$

Since $Q_{0}=S_{0}>0$, relations (2.17) imply that there is a limit

$$
\lim _{k \rightarrow \infty} Q_{k}^{-1}=\varkappa_{Q} \geq 0 \text {. }
$$

\subsection{Skew-self-adjoint case}

The preliminary definitions and results on the skew-self-adjoint discrete Dirac systems (SkDDS) (1.5) we take from [6] and sometimes from [8].

Remark 2.3 The notations here slightly differ from the notations in [6, 8]. In particular, we introduce the matrices $R_{k}$ and $Q_{k}$ in the both self-adjoint and skew-self-adjoint cases via formulas (2.13) and (2.16), respectively, but in [8] $R_{k}$ stands for $Q_{k}$ in the the present notations and $Q_{k}$ stands for $R_{k}$.

Definition 2.4 The Weyl function of $S k D D S$ is an $m_{1} \times m_{2}$ matrix function $\varphi(z)$ in

$$
\mathbb{C}_{M}=\{z \in \mathbb{C}: \Im(z)>M\} \text { for some } M>0,
$$

which satisfies the inequality

$$
\sum_{k=0}^{\infty}\left[\varphi(z)^{*} \quad I_{m_{2}}\right] w_{k}(z)^{*} w_{k}(z)\left[\begin{array}{c}
\varphi(z) \\
I_{m_{2}}
\end{array}\right]<\infty,
$$

where $w_{k}(z)$ is the fundamental solution of SkDDS normalized by $w_{0}(z) \equiv I_{m}$. 
Let us fix again an integer $n>0$, and consider an $n \times n$ matrix $A$ with $\operatorname{det} A \neq 0$, an $n \times n$ matrix $S_{0}>0$ and an $n \times m$ matrix $\Pi_{0}$. These matrices should satisfy the identity

$$
A S_{0}-S_{0} A^{*}=\mathrm{i} \Pi_{0} \Pi_{0}^{*}
$$

The sequences $\left\{\Pi_{k}\right\},\left\{S_{k}\right\}$ and $\left\{C_{k}\right\}(k \geq 0)$ are introduced using the triple $\left\{A, S_{0}, \Pi_{0}\right\}$ and relations

$$
\begin{aligned}
& \Pi_{k+1}=\Pi_{k}+\mathrm{i} A^{-1} \Pi_{k} j, \\
& S_{k+1}=S_{k}+A^{-1} S_{k}\left(A^{*}\right)^{-1}+A^{-1} \Pi_{k} j \Pi_{k}^{*}\left(A^{*}\right)^{-1}, \\
& C_{k}=j+\Pi_{k}^{*} S_{k}^{-1} \Pi_{k}-\Pi_{k+1}^{*} S_{k+1}^{-1} \Pi_{k+1} .
\end{aligned}
$$

Similar to the self-adjoint case we write down $\Pi_{k}$ in the form

$$
\Pi_{k}=\left[\begin{array}{ll}
\left(I_{n}+\mathrm{i} A^{-1}\right)^{k} \vartheta_{1} & \left(I_{n}-\mathrm{i} A^{-1}\right)^{k} \vartheta_{2}
\end{array}\right] .
$$

If $S_{0}>0$, the identity (2.20) holds and the pair $\left\{A, \vartheta_{1}\right\}$ is controllable, then according to [6. Lemma 3.2] and [6, Proposition 3.6] we have $\operatorname{det} A \neq 0$, $S_{k}>0$ and the matrices $C_{k}$ admit representation $C_{k}=U_{k}^{*} j U_{k}$ from (1.5). That is, the sequence $\left\{C_{k}\right\}$ is well-defined and the corresponding system is a skew-self-adjoint Dirac system. In the skew-self-adjoint case, the triple $\left\{A, S_{0}, \Pi_{0}\right\}$, such that $S_{0}>0$, the identity (2.20) holds and the pair $\left\{A, \vartheta_{1}\right\}$ is controllable, is called admissible. The potential determined by this triple is called pseudo-exponential.

Moreover, if $\varphi(z)$ is a strictly proper rational $m_{1} \times m_{2}$ matrix function then it is the Weyl function of some skew-self-adjoint Dirac system with the pseudo-exponential potential (see [8, Theorem 4.2]). We will require additionally that $\mathrm{i} \notin \sigma(A)$.

Definition 2.5 In the skew-self-adjoint case, the triple $\left\{A, S_{0}, \Pi_{0}\right\}$, where $S_{0}>0$, the identity (2.20) is valid, the pair $\left\{A, \vartheta_{1}\right\}$ is controllable and $\mathrm{i} \notin \sigma(A)$, is called strongly admissible. The potentials determined by the strongly admissible triples are called strictly pseudo-exponential.

Note that [8, Proposition 4.8] implies that if $S_{0}>0$, (2.20) holds and 0 , i $\notin \sigma(A)$ then $S_{k}>0$, the matrices $C_{k}$ are well-defined and there is 
a strongly admissible triple which determines the same potential $\left\{C_{k}\right\}$ as $\left\{A, S_{0}, \Pi_{0}\right\}$. The fundamental solution $w_{k}$ of SkDDS determined by the strongly admissible triple $\left\{A, S_{0}, \Pi_{0}\right\}$ has the form

$$
w_{k}(z)=w_{A}(k,-z)\left(I_{m}+\frac{\mathrm{i}}{z} j\right)^{k} w_{A}(0,-z)^{-1},
$$

whereas $w_{A}$ in the skew-self-adjoint case is given by

$$
w_{A}(k, \lambda):=I_{m}-\mathrm{i} \Pi_{k}^{*} S_{k}^{-1}\left(A-\lambda I_{n}\right)^{-1} \Pi_{k} .
$$

Taking into account Remark [2.3, we see that [8, Proposition 4.10] and [8, (4.34)] imply that

$$
\lim _{k \rightarrow \infty} Q_{k}^{-1}=0 ; \quad \lim _{k \rightarrow \infty}\left(Q_{k}^{-1} \widetilde{G}(A)^{k} \vartheta_{1}\right)=0, \quad \widetilde{G}(A):=\left(A-\mathrm{i} I_{n}\right)^{-1}\left(A+\mathrm{i} I_{n}\right)
$$

in the case of a strongly admissible triple $\left\{A, S_{0}, \Pi_{0}\right\}$.

\section{Reflection coefficients}

\subsection{Reflection coefficients: self-adjoint case}

In this subsection, we express (via the triple $\left\{A, S_{0}, \Pi_{0}\right\}$ ) the Jost solution and reflection coefficient, which are the analogs of the corresponding functions in the continuous case.

Uniqueness of the solution of the inverse problem to recover system from the Weyl function (see [18, Theorem 2.3]) together with Theorems 2.6 and 2.8 and Proposition 2.7 (all from [18]) imply that without loss of generality one can require that $\sigma(A) \subset\left(\mathbb{C}_{+} \cup \mathbb{R}\right)$. Further we use a stronger requirement

$$
\sigma(A) \subset \mathbb{C}_{+}, \quad \mathrm{i} \notin \sigma(A)
$$

Following [6, 10, we call the admissible triple satisfying (3.1) strongly admissible and we introduce the class of the strictly pseudo-exponential potentials $\left\{C_{k}\right\}$. 
Definition 3.1 The potentials $\left\{C_{k}\right\}$ of the Dirac systems (1.1), (1.2), which are determined by the strongly admissible triples, are called strictly pseudoexponential.

In view of (2.9), (2.11), (2.13) and (2.16), we have a representation

$$
\begin{aligned}
& w_{A}(k,-1 / z) \\
& =I_{m}-\mathrm{i} z j\left[\begin{array}{cc}
\vartheta_{1}^{*} R_{k}^{-1}\left(I_{n}+z A\right)^{-1} \vartheta_{1} & \vartheta_{1}^{*} R_{k}^{-1}\left(I_{n}+z A\right)^{-1} G(A)^{k} \vartheta_{2} \\
\vartheta_{2}^{*}\left(G(A)^{k}\right)^{*} R_{k}^{-1}\left(I_{n}+z A\right)^{-1} \vartheta_{1} & \vartheta_{2}^{*} Q_{k}^{-1}\left(I_{n}+z A\right)^{-1} \vartheta_{2}
\end{array}\right],
\end{aligned}
$$

where

$$
G(A)=\left(I_{n}+\mathrm{i} A^{-1}\right)^{-1}\left(I_{n}-\mathrm{i} A^{-1}\right) .
$$

Relations (3.1) and (3.3) yield

$$
\sigma(G(A)) \subset\{\lambda:|\lambda|<1\} .
$$

Hence, from (2.15), (2.18) and (3.2) we derive

$$
\begin{aligned}
& \lim _{k \rightarrow \infty} w_{A}(k,-1 / z)=\left[\begin{array}{cc}
\chi_{1}(z) & 0 \\
0 & \chi_{2}(z)
\end{array}\right], \\
& \chi_{1}(z):=I_{m_{1}}-\mathrm{i} z \vartheta_{1}^{*} \varkappa_{R}\left(I_{n}+z A\right)^{-1} \vartheta_{1}, \chi_{2}(z):=I_{m_{2}}+\mathrm{i} z \vartheta_{2}^{*} \varkappa_{Q}\left(I_{n}+z A\right)^{-1} \vartheta_{2} .
\end{aligned}
$$

According to (1.3), (2.10) and (3.5), the Jost solution $\left\{F_{k}\right\}$ is given by the equalities

$$
F_{k}(z)=W_{k}(z) w_{A}(0,-1 / z)\left[\begin{array}{cc}
\chi_{1}(z)^{-1} & 0 \\
0 & \chi_{2}(z)^{-1}
\end{array}\right] .
$$

Partition $w_{A}$ into the blocks corresponding to the partitioning of $j$ :

$$
w_{A}(0, \lambda)=\left[\begin{array}{ll}
a(\lambda) & b(\lambda) \\
c(\lambda) & d(\lambda)
\end{array}\right] .
$$

It was shown in the proof of [18, Theorem 2.6] (see [18, (2.29)]) that the Weyl function $\varphi(z)$ of the system (1.1), (1.2) (in $\mathbb{C}_{-}$) is given by the formula

$$
\varphi(z)=b(-1 / z) d(-1 / z)^{-1} .
$$

Relations (1.4), (2.1) and (3.7)-(3.9) imply the following theorem. 
Theorem 3.2 Let Dirac system (1.1), (1.2) be a system with the strictly pseudo-exponential potential $\left\{C_{k}\right\}$. Then the Weyl function $\varphi(z)$ is the unique analytic continuation of the reflection coefficient $\mathcal{R}(z)$ of this system. That is, the reflection coefficient and the Weyl function are given by the same rational matrix function.

From [18, Theorem 2.6] and Theorem 3.2 we derive the following corollary.

Corollary 3.3 Let the potential $\left\{C_{k}\right\}$ be determined by a strongly admissible triple $\left\{A, S_{0}, \Pi_{0}\right\}$. Then, the reflection coefficient of the Dirac system (1.1), (1.2) is given by the formula

$$
\mathcal{R}(z)=-\mathrm{i} z \vartheta_{1}^{*} S_{0}^{-1}\left(I_{n}+z A^{\times}\right)^{-1} \vartheta_{2}, \quad A^{\times}=A+\mathrm{i} \vartheta_{2} \vartheta_{2}^{*} S_{0}^{-1} .
$$

\subsection{Reflection coefficients: skew-self-adjoint case}

In the skew-self-adjoint case, we define the reflection coefficient $\mathcal{R}(z)$ in a slightly more general way than in the self-adjoint case. That is, we consider the matrix valued $m_{2} \times m$ solution $Y$ of the system (1.5)):

$$
Y_{k}(z)=\left(1-\frac{\mathrm{i}}{z}\right)^{k}\left(\left[\begin{array}{c}
0 \\
I_{m_{2}}
\end{array}\right]+o(1)\right), \quad k \rightarrow \infty
$$

and set

$$
\mathcal{R}(z)=\left[\begin{array}{ll}
I_{m_{1}} & 0
\end{array}\right] Y_{0}(z)\left(\left[\begin{array}{ll}
0 & I_{m_{2}}
\end{array}\right] Y_{0}(z)\right)^{-1} .
$$

In order to express $\mathcal{R}(z)$ via a strongly admissible triple $\left\{A, S_{0}, \Pi_{0}\right\}$, we derive from (2.11), (2.13), (2.16) and (2.25) the representation

$$
w_{A}(k,-z)\left[\begin{array}{c}
0 \\
I_{m_{2}}
\end{array}\right]=\left[\begin{array}{c}
0 \\
I_{m_{2}}
\end{array}\right]-\mathrm{i}\left[\begin{array}{c}
\vartheta_{1}^{*}\left(\widetilde{G}(A)^{k}\right)^{*} Q_{k}^{-1}\left(z I_{n}+A\right)^{-1} \vartheta_{2} \\
\vartheta_{2}^{*} Q_{k}^{-1}\left(z I_{n}+A\right)^{-1} \vartheta_{2}
\end{array}\right],
$$

where $\widetilde{G}$ is introduced in (2.26). Formulas (2.26) and (3.13) imply that

$$
\lim _{k \rightarrow \infty} w_{A}(k,-z)\left[\begin{array}{c}
0 \\
I_{m_{2}}
\end{array}\right]=\left[\begin{array}{c}
0 \\
I_{m_{2}}
\end{array}\right] .
$$


It follows from (2.24), (3.11) and (3.14) that

$$
Y_{k}(z)=\left(1-\frac{\mathrm{i}}{z}\right)^{k} w_{A}(k,-z)\left[\begin{array}{c}
0 \\
I_{m_{2}}
\end{array}\right] .
$$

Hence, after we take into account (3.12) and (similar to the self-adjoint case) partition $w_{A}$ (as in (3.8)), we obtain

$$
\mathcal{R}(z)=b(-z) d(-z)^{-1}
$$

On the other hand, according to [6, (3.24)] the Weyl function $\varphi(z)$ of the system (1.5) is also given by the right-hand side of (3.16). Thus, the following theorem is proved.

Theorem 3.4 Let Dirac system (1.5) be a system with the strictly pseudoexponential potential $\left\{C_{k}\right\}$. Then the Weyl function $\varphi(z)$ is the analytic continuation of the reflection coefficient $\mathcal{R}(z)$ of this system. More precisely, the reflection coefficient and the Weyl function are given by the same rational matrix function.

The next corollary follows from [6, Theorem 3.8] and Theorem 3.4.

Corollary 3.5 Let the potential $\left\{C_{k}\right\}$ be determined by a strongly admissible triple $\left\{A, S_{0}, \Pi_{0}\right\}$. Then, the reflection coefficient of the skew-self-adjoint Dirac system (1.5) is given by the formula

$$
\mathcal{R}(z)=-\mathrm{i} \vartheta_{1}^{*} S_{0}^{-1}\left(z I_{n}+z A^{\times}\right)^{-1} \vartheta_{2}, \quad A^{\times}=A-\mathrm{i} \vartheta_{2} \vartheta_{2}^{*} S_{0}^{-1} .
$$

Acknowledgments. The research of A.L. Sakhnovich was supported by the Austrian Science Fund (FWF) under Grant No. P29177.

\section{References}

[1] J. Behrndt, M.M. Malamud, and H. Neidhardt, Scattering matrices and Weyl functions, Proc. Lond. Math. Soc. 97 (2008), pp. 568-598.

[2] J.L. Cieslinski, Algebraic construction of the Darboux matrix revisited, J. Phys. A 42 (2009), 404003. 
[3] S. Clark and F. Gesztesy, On Weyl-Titchmarsh theory for singular finite difference Hamiltonian systems, J. Comput. Appl. Math. 171 (2004), pp. 151-184.

[4] P.A. Deift, Applications of a commutation formula, Duke Math. J. 45 (1978), pp. 267-310.

[5] M. Derevyagin and B. Simanek, Szegö's theorem for a nonclassical case, J. Funct. Anal. 272 (2017), pp. 2487-2503.

[6] B. Fritzsche, M.A. Kaashoek, B. Kirstein, and A.L. Sakhnovich, Skew-selfadjoint Dirac systems with rational rectangular Weyl functions: explicit solutions of direct and inverse problems and integrable wave equations, Math. Nachr. 289 (2016), pp. 1792-1819.

[7] B. Fritzsche, B. Kirstein, I. Ya. Roitberg, and A.L. Sakhnovich, Discrete Dirac system: rectangular Weyl, functions, direct and inverse problems, Oper. Matrices 8:3 (2014), pp. 799-819.

[8] B. Fritzsche, B. Kirstein, I.Ya. Roitberg, and A.L. Sakhnovich, Stability of the procedure of explicit recovery of skew-selfadjoint Dirac systems from rational Weyl matrix functions, Linear Algebra Appl. 533 (2017), pp. 428-450.

[9] F. Gesztesy and G. Teschl, On the double commutation method, Proc. Amer. Math. Soc. 124 (1996), pp. 1831-1840.

[10] I. Gohberg, M.A. Kaashoek, and A.L. Sakhnovich, Canonical systems with rational spectral densities: explicit formulas and applications, Math. Nachr. 194 (1998), pp. 93-125.

[11] I. Gohberg, M.A. Kaashoek, and A.L. Sakhnovich, Scattering problems for a canonical system with a pseudo-exponential potential, Asymptotic Analysis 29 (2002), pp. 1-38.

[12] C. Gu, H. Hu, and Z. Zhou, Darboux Transformations in Integrable Systems, Springer, Dordrecht, 2005. 
[13] J. Janas and S.A. Simonov, Weyl-Titchmarsh type formula for a discrete Schrödinger operator with Wigner-von Neumann potential, Studia Math. 201 (2010), pp. 167-189.

[14] M.A. Kaashoek and A.L. Sakhnovich, Discrete skew self-adjoint canonical system and the isotropic Heisenberg magnet model, J. Funct. Anal., 228 (2005), pp. 207-233.

[15] A. Kostenko, A. Sakhnovich, and G. Teschl, Commutation methods for Schrödinger operators with strongly singular potentials, Math. Nachr. 285:4 (2012), pp. 392-410.

[16] V.A. Marchenko, Nonlinear Equations and Operator Algebras, D. Reidel, Dordrecht, 1988.

[17] V.B. Matveev and M.A. Salle, Darboux transformations and solitons. Springer, Berlin, 1991.

[18] I.Ya. Roitberg and A.L. Sakhnovich, General-type discrete selfadjoint Dirac systems: explicit solutions of direct and inverse problems, asymptotics of Verblunsky-type coefficients and stability of solving inverse problem, arXiv:1802.10557 (J. Math. Phys. Anal. Geom., issue dedicated to V.A. Marchenko, 2018 to appear).

[19] A.L. Sakhnovich, Dressing procedure for solutions of nonlinear equations and the method of operator identities, Inverse Problems 10:3 (1994), pp. 699-710.

[20] A.L. Sakhnovich, Toeplitz matrices with an exponential growth of entries and the first Szegö limit theorem, J. Funct. Anal. 171 (2000), pp. 449-482.

[21] A.L. Sakhnovich, Generalized Bäcklund-Darboux transformation: spectral properties and nonlinear equations, J. Math. Anal. Appl. 262:1 (2001), pp. 274-306.

[22] A.L. Sakhnovich, Verblunsky-type coefficients for Dirac and canonical systems generated by Toeplitz and Hankel matrices, respectively, arXiv:1711.03064. 
[23] A.L. Sakhnovich, Scattering for general-type Dirac systems on the semi-axis: reflection coefficients and Weyl functions, arXiv:1801.10020

[24] A.L. Sakhnovich, L.A. Sakhnovich, and I.Ya. Roitberg, Inverse Problems and Nonlinear Evolution Equations. Solutions, Darboux Matrices and Weyl-Titchmarsh Functions, De Gruyter Studies in Mathematics Vol. 47, De Gruyter, Berlin, 2013.

[25] L.A. Sakhnovich, On the factorization of the transfer matrix function, Sov. Math. Dokl. 17 (1976), pp. 203-207.

[26] L.A. Sakhnovich, Spectral Theory of Canonical Differential Systems, Method of Operator Identities, Operator Theory Adv. Appl. Vol. 107, Birkhäuser Verlag, Basel, 1999.

[27] R. Simon Hilscher and P. Zemanek, Weyl-Titchmarsh theory for discrete symplectic systems with general linear dependence on spectral parameter, J. Difference Equ. Appl. 20 (2014), pp. 84-117.

[28] P. Zemanek and S. Clark, Characterization of self-adjoint extensions for discrete symplectic systems, J. Math. Anal. Appl. 440 (2016), pp. 323-350. 
B. Fritzsche, Fakultät für Mathematik und Informatik, Universität Leipzig, Augustusplatz 10, D-04009 Leipzig, Germany, e-mail: Bernd.Fritzsche@math.uni-leipzig.de

B. Kirstein, Fakultät für Mathematik und Informatik, Universität Leipzig, Augustusplatz 10, D-04009 Leipzig, Germany, e-mail: Bernd.Kirstein@math.uni-leipzig.de

I.Ya. Roitberg, e-mail: innaroitberg@gmail.com

A.L. Sakhnovich, Faculty of Mathematics, University of Vienna, Oskar-Morgenstern-Platz 1, A-1090 Vienna, Austria, e-mail: oleksandr.sakhnovych@univie.ac.at 